Ciencias Agrarias/Agrarian Sciences

Cienc Tecn UTEQ (2016) 9(2) p 11-17 ISSN 1390-4051; e-ISSN 1390-4043 (9)(3)

\title{
Comercialización de arroz en Ecuador: Análisis de la evolución de precios en el eslabón productor-consumidor
}

\section{Sales prices of rice in Ecuador producer - consumer and their correlation links in the production chain}

\author{
${ }^{\bullet}$ Gladys Irene Viteri Viteri ${ }^{1}$, Carlos Edison Zambrano ${ }^{2,3}$ \\ ${ }^{1}$ Instituto Nacional de Investigaciones Agropecuarias-INIAP, Estación Experimental del Litoral Sur. Departamento de \\ Planificación y Economía Agrícola. Km 26 Via Durán-Tambo, al Oeste de Guayaquil, Cantón Yaguachi, Guayas. Ecuador. \\ gladys.viteri@iniap.gob.ec \\ ${ }^{2}$ Universidad Técnica Estatal de Quevedo. Facultad de Ciencias Empresariales, Carrera de Economía. Campus \\ “Ingeniero Manuel Haz Álvarez”, Av. Quito, km 1.5 vía a Santo Domingo de los Tsáchilas. EC.120301. Quevedo, Ecuador. \\ cezambrano@uteq.edu.ec \\ ${ }^{3}$ Instituto Nacional de Investigaciones Agropecuarias-INIAP. Estación Experimental Tropical Pichilingue. Departamento de \\ Planificación y Economía Agrícola. Km 5 1/2 vía Quevedo-El Empalme, Cantón Mocache, Los Ríos. Ecuador. \\ carlos.zambrano@iniap.gob.ec
}

Rec.: 6.04.2016. Acept.: 8.07.2016.

Publicado el 30 de diciembre de 2016

Resumen

$\mathrm{E}_{\mathrm{la}}^{\mathrm{n}}$ Ecuador el arroz tiene importancia económica y social, la superficie sembrada en el año 2013 fue de 414,096 hectáreas, con $93.94 \%$ en las provincias de Guayas y Los Ríos. La cadena genera un movimiento económico superior a los 146 millones de dólares que se inicia con el productor, luego el acopiador rural, pilador y/o agroindustrial, los comerciantes mayorista y minorista y al final el consumidor. El objetivo de la investigación fue analizar la evolución de precios de arroz en cáscara (paddy) y pilado en los eslabones productorconsumidor y su correlación durante el periodo 2000-2013. El análisis de datos se realizó con Stat graphics para variables individuales, mediante estimación de ecuaciones de regresión y correlación. Durante los años de estudio la variable individual, precios recibidos por el productor de arroz en cáscara en promedio fue de USD $0.23 \mathrm{~kg}^{-1}$, con un rango entre USD 0.12 y USD $0.35 \mathrm{~kg}^{-1}$, mientras que la variable precios por kilogramo de arroz pilado a nivel del consumidor, registró en promedio USD 0.75 , con un rango entre USD 0.47 y USD $1.05 \mathrm{~kg}^{-1}$. Se concluye que los precios del productor están directamente relacionados con el precio al consumidor y éstos a su vez se relacionan con el consumo per cápita, por lo que se realizó un pronóstico de precios para los próximos 12 años.

Palabras clave: consumo per cápita, precio al productor, precio al consumidor, arroz paddy, pronóstico de precios.
I n Ecuador, rice has economic and social importance, the area planted in 2013 was 414,096 hectares, with $93.94 \%$ in the provinces of Guayas and Los Rios. The chain, which begins with the producer, then the striker, pilador and / or agro-industry, the wholesaler, the retailer, and end consumers generates a more than $\$ 146$ million economic movement. The aim of the research was to analyze the evolution of paddy and milled rice prices in the producer-consumer links and their correlation over the period 2000-2013. Data analysis was performed using the Stat graphics for individual variables by estimating regression and correlation equations. During the years of study individual variable, prices received by paddy rice producers was USD $0.23 \mathrm{~kg}^{-1}$ average, with a range between USD 0.12 and USD $0.35 \mathrm{~kg}^{-1}$, while the variable price per milled rice kilogram at the consumer level recorded, on average, USD 0.75 , with a range between USD 0.47 and USD $1.05 \mathrm{~kg}^{-1}$. It is concluded that producer prices are directly linked to the consumer price and these, in turn, are related to the level of consumption, so a price forecast for the next 12 years was performed.

Key words: consumption per capita, producer price, consume price, rice paddy, price forecast. 


\section{Introducción}

$\mathrm{E}$ arroz Oryza sativa L, es el cereal más importante del mundo en desarrollo, constituye el alimento básico para más de la mitad de la población del planeta (Briceño y Álvarez, 2010). En Ecuador el cultivo de arroz es la principal fuente alimenticia, formando parte de la dieta básica de los habitantes de la Costa ecuatoriana (Garcés et al., 2012); de hecho, los 53.20 kilogramos por habitante de consumo anual definen la magnitud de su importancia frente a países vecinos como Colombia y Perú que consumen anualmente 40.0 y $47.4 \mathrm{~kg}$ por habitante, respectivamente (Gavilánez et al., 2016).

Los productos del sector agroalimentario tienen una característica principal, la volatilidad de los precios, debido a varios factores, entre ellos, oferta, demanda, crecimiento de la población, variables biológicas y fenómenos naturales (Ceballos y Pire, 2015), por lo que la producción de arroz, requiere de análisis integrales a fin de mejorar su eficiencia y competitividad (Najar y Álvarez, 2007). La evolución de precios de los bienes básicos (commodities) ha sido una de las mayores preocupaciones para los responsables de la política económica en los últimos años por su influencia en la dinámica de la actividad económica, la inflación y la balanza de pagos (Arteaga et al., 2013).

$\mathrm{El}$ arroz que se produce es fruto no solo de la fortuna de las características del suelo y clima, sino de una serie de procesos coyunturales, endógenos y organizativos entre arroceros y diversas instituciones locales a través de los años (Tolentino, 2014). La cadena arrocera es constituida por los productores de arroz, que realizan la fase de producción de materia prima (arroz en cáscara verde) y las pequeñas, medianas y grandes agroindustrias arroceras responsables del procesamiento agroindustrial y la distribución del producto a consumidores (Vivas y Albisu, 2011).

En Ecuador, la superficie sembrada con arroz en el año 2013 fue de 414.096 hectáreas, con una producción de 1515,836 t (SINAGAP, 2015), siendo Guayas y Los Ríos las provincias que aportan con el $93.79 \%$, el resto se distribuye en otras provincias. En el mercado mundial, el precio de arroz por tonelada métrica en el 2013, en promedio fue de US $\$ 518.81 \mathrm{t}^{-1}$, con fluctuación entre US $\$ 447 \mathrm{t}^{-1}$ y US $\$ 574$ $\mathrm{t}^{-1}$, inferior al del 2012 cuyo promedio fue de $580.24 \mathrm{t}^{-1}$ (Indexmundi, 2015).

En Ecuador el mayor porcentaje de la producción de arroz se comercializa como grano en cáscara durante los meses de abril a mayo y unos pocos lo venden pilado. En el canal de distribución hay intermediarios que modifican los precios, ampliando el margen de diferencia entre lo que paga el consumidor final y lo que ha recibido el productor (Alonso y Gallego, 2010). Adicionalmente es importante destacar la vulnerabilidad de la cadena en relación a la dependencia del Estado para la comercialización de arroz (Vieira et al., 2014). La Asamblea Nacional (2011), introdujo un mecanismo de precios a 46 productos agropecuarios incluido el arroz, por lo que el MAGAP (2014) dispuso mantener el precio mínimo de sustentación de la saca de arroz cáscara de $90.72 \mathrm{~kg}$, con $20 \%$ de humedad y $5 \%$ de impurezas para la campaña agrícola 2014 en USD 34.50. El precio a nivel de consumidor lo regula la oferta y demanda que oscila entre 45 y 52 dólares el quintal lo que representa entre 0.99 y 1.16 dólares por kilogramo.

Los precios agrícolas a nivel internacional han sido evaluados a través de modelos de series temporales y de causalidad, sin embargo, la literatura científica disponible sobre análisis del mercado de arroz ecuatoriano es limitada por no decir inexistente por lo que esta investigación tuvo por objetivo analizar la evolución de precios de arroz en cáscara y pilado en los eslabones productor-consumidor durante el periodo 2000-2013 y al mismo tiempo proponer una herramienta estadística para predecir los precios. Se partió de la hipótesis que los consumidores resultan perjudicados por la evolución de los precios de arroz debido a que coexisten situaciones de precios bajos en origen con altos precios en destino.

\section{Materiales y métodos}

Ta investigación se realizó en la Estación Experimental del Litoral Sur del INIAP, ubicada al Este de Guayaquil, km. 26 de la carretera Durán Tambo, cantón Yaguachi. Longitud $79^{\circ} .5 \mathrm{~W}$ y latitud $2^{\circ} .3 \mathrm{~S}$; a $17 \mathrm{msnm}$, su temperatura media anual oscila entre los $22^{\circ} \mathrm{C}$ y $25^{\circ} \mathrm{C}$; precipitación anual promedio de 1,398 milímetros. Para evaluar los precios de arroz a nivel de productor y consumidor se utilizaron datos del MAGAP (2015), igualmente la misma institución proporcionó la información estadística de costos de producción y consumo per cápita.

Se ajustaron modelos lineales con series de tiempo del 2000 al 2013, mediante un modelo matemático relacionando la variable dependiente $\mathrm{Y}$ con la variable independiente $\mathrm{X}$ cuya función lineal es $\mathrm{Y}=\mathrm{a}+\mathrm{bX}$. Como medida de bondad del ajuste se utilizó el coeficiente de determinación $\mathrm{R}^{2}=\mathrm{scE} / \mathrm{scG}$ y mediante análisis de varianza se estableció el grado de correlación entre los precios del productor con los del consumidor. Además, se realizaron pronósticos de comportamiento de precios a nivel de productor y consumidor para los próximos 12 años, asumiendo que los posibles cambios en la oferta y la demanda derivados, por ejemplo, de cambios en el mercado internacional o doméstico, población, clima, entre otras; están sujetas a las condiciones establecidas en el cálculo del modelo de regresión del período 2000 al 2013. 


\section{Resultados y discusión}

\section{Precio a nivel del productor}

En promedio, a cada incremento de una unidad en el precio por kilogramo de arroz le corresponde un incremento de 0.0934 dólares por año. La variable individual, precios al productor revela que en promedio fue de USD 0.23 , con rango entre USD 0.12 y USD $0.37 \mathrm{~kg}^{-1}$ de arroz en cáscara, aumentándose en $146.7 \%$ entre el año 2000 y 2013. Se observa que el precio al productor se incrementa a través del tiempo en USD 0.0191. Además, revela que en los 14 años analizados se registran oscilaciones en los precios que están relacionados con la variación en la cantidad cosechada por problemas de tipo bióticos como ataque del molusco (Pomacea canaliculata) y enfermedades como hoja blanca a consecuencia del abuso de plaguicidas para el manejo del caracol; también patógenos del suelo, complejo del manchado del grano, manejo inadecuado del cultivo (INIAP, 2013) y posible efecto del cambio climático ya que según Monasterio et al., (2011) durante los años 2000 al 2010 se presentaron al menos 4 fenómenos de La Niña y 5 fenómenos de El Niño, lo que ha ocasionado variaciones en la productividad (Figura 1).
El pronóstico de precios al productor de arroz en cáscara, para los próximos 12 años (Figura 2), demuestra que fluctuará entre USD 0.40 y 0.50 con límite inferior entre USD 0.34 y 0.39 y superior entre USD 0.46 y $0.61 \mathrm{~kg}^{-1}$. Basados en la proyección, deberían supervisarse los precios oficiales en los eslabones de la cadena, especialmente a los productores que en su mayoría venden a las piladoras e intermediarios; por el temor de entregar a la Unidad Nacional de Almacenamiento (UNA), porque el proceso de selección y calificación del grano es estricto al momento de la venta (BCE, 2015). A partir del año 2008 el gobierno viene fijando precios piso y techo para el arroz en cáscara en sacas de 200 libras $(90.70 \mathrm{~kg}$ ) con $20 \%$ de humedad y $5 \%$ de impurezas los mismos que siempre están al alza lo cual también contribuye al incremento de precios al productor durante el período analizado.

\section{Precio a nivel del consumidor}

Los resultados de ésta variable, señalan que el precio promedio por $\mathrm{kg}$ de arroz pilado fue de USD 0.75 , con mínimo de USD 0.47 y máximo de $1.05 \mathrm{~kg}^{-1}$. La ecuación de regresión y el coeficiente de determinación obtenido brindan confiabilidad al modelo (Figura 3). El precio del arroz para las familias se ha mantenido al alza, observándose un incremento

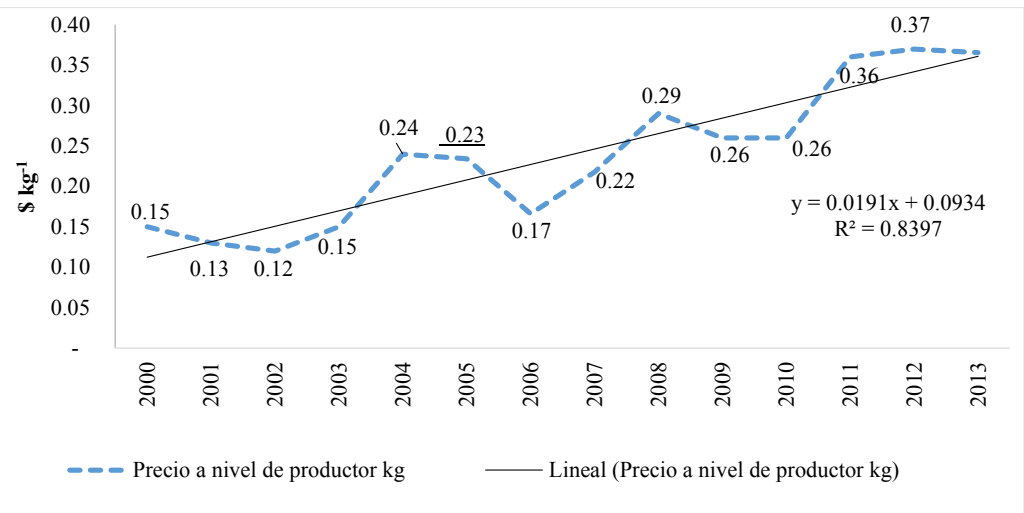

Figura 1. Precio a nivel productor por kg de arroz en cáscara, periodo 2000-2013

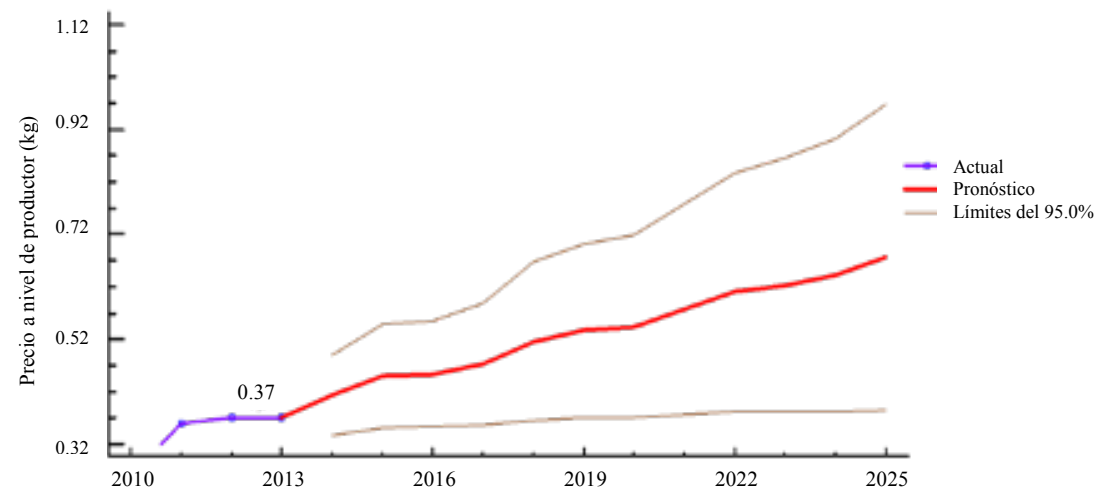

Figura 2. Pronóstico para precios a nivel productor de arroz en cáscara $\left(\$ \mathrm{~kg}^{-1}\right)$, periodo $2014-2025$ 


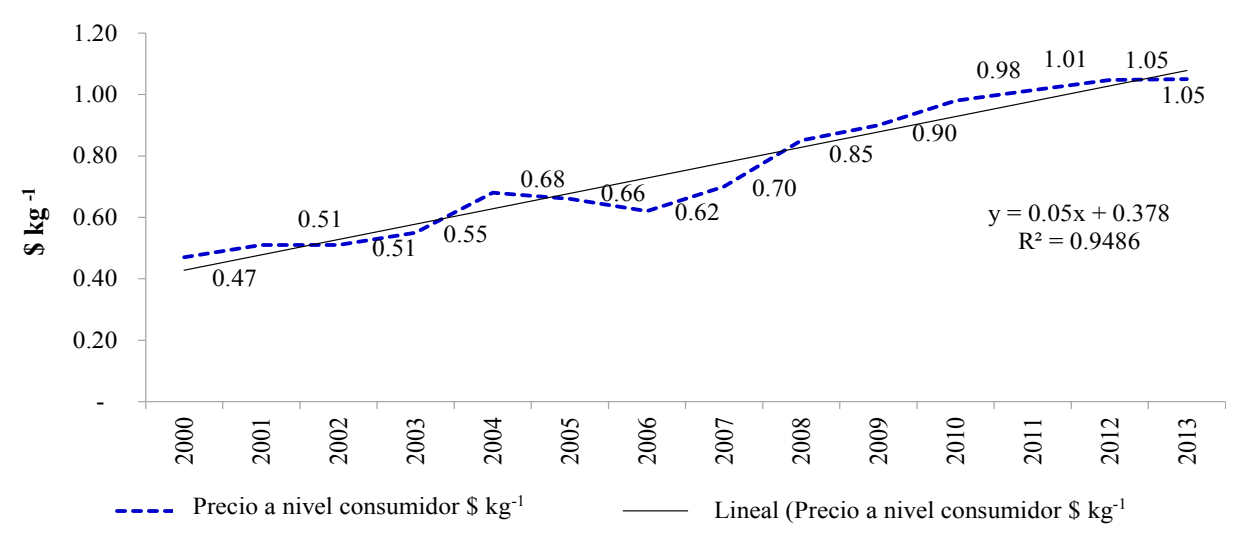

Figura 3. Precios a nivel consumidor, por kilogramo de arroz pilado, periodo 2000-2013

del $123.4 \%$ del año 2000 al 2013 pese a los precios oficiales. En el comportamiento de los precios al consumidor es necesario destacar la tendencia a la baja en los precios de arroz para el año 2005 y especialmente del año 2006 provocada por la sobre oferta de la gramínea lo cual influenció para alcanzar un récord de exportaciones de 156,505 t durante éste último año.

Durante los años 2007 y 2008 el precio de arroz al consumidor registró el mayor incremento del período, básicamente por la presión ejercida en los mercados locales de comerciantes acopiadores-exportadores de la gramínea hacia Colombia. El análisis de pronóstico de precios al consumidor para los próximos 12 años (Figura 4), indica que el precio al consumidor alcanzará valores entre USD 1.13 y $1.38 \mathrm{~kg}$ con límites; inferior de USD 1.00 y 1.23 y superior entre USD 1.25 y 1.52 .

Pese a que los precios de arroz a nivel de consumidor tienen una tendencia hacia arriba, en estudios de mercado se ha demostrado que los consumidores de arroz buscan en primer lugar, calidad del producto en el momento de decidir su compra, por lo que no es el precio el factor determinante para su decisión (Cortez y Vargas, 2013).

\section{Precio del productor versus precio del consumidor}

El análisis de varianza muestra una correlación positiva significativa con $\mathrm{r}=0.9476$ y un $\mathrm{p}<0.05$, entre el precio del productor con el consumidor. Si bien la evolución de precios del productor guardan correlación con los precios al consumidor, se nota una diferencia en la variable precios al productor, con un promedio de USD 0.23 , frente al precio superior promedio por $\mathrm{kg}$ de arroz al consumidor que fue de USD 0.75 , confirmándose la hipótesis de que coexisten situaciones de precios bajos en origen con altos precios en destino. Una conducta similar ocurrió con los precios agrarios percibidos y pagados por el agricultor en España durante el período 2000-2009, según lo reportado por Andrés et al. (2012), los agricultores perciben bajos precios por sus productos, pero los precios de los consumidores finales no están acordes con dichos precios.

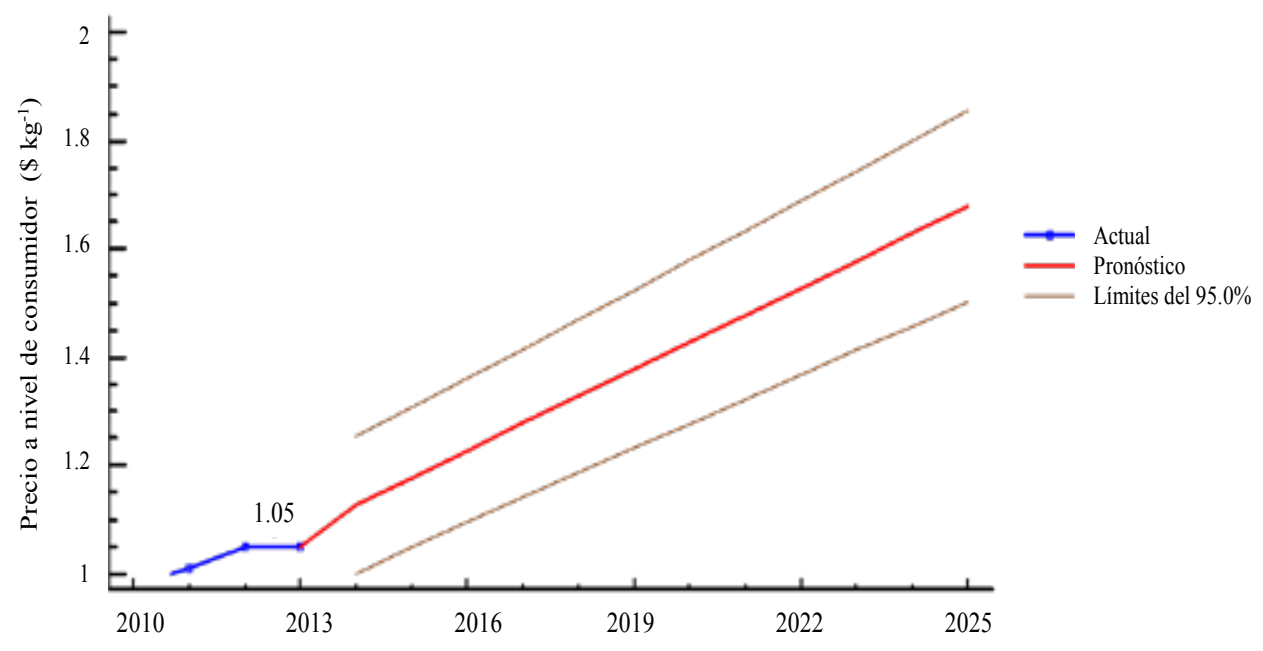

Figura 4. Pronóstico para precio de arroz a nivel consumidor $\left(\$ \mathrm{~kg}^{-1}\right)$, periodo 2014-2025 


\section{Precio del productor versus costo de producción}

En estas variables se obtuvo un coeficiente de correlación lineal de $0.905(\mathrm{p}<0.05)$; siendo la desviación estándar de los residuos 0.03866 , que puede usarse para construir límites de predicción para nuevas observaciones.

Los precios a nivel de productor (Figura 5) fueron superiores a los costos por $\mathrm{kg}$ producido durante ocho años (2000, 2004, 2005, 2007, 2008, 2011, 2012, 2013), mientras, los otros seis años, los precios de venta fueron inferiores o iguales a los costos de producción.

Los datos de mejores precios son en parte explicados por el accionar de la Unidad Nacional de Almacenamiento (UNA), creada en el año 2007, que se consolidó en Empresa Pública UNA-EP en el año 2013 y viene controlando el pago de precios mínimos de sustentación de arroz en beneficio de los agricultores, aplicando tres factores: cobertura (cinco plantas de silos fijos, 14 centros de acopio de arroz); capacidad de almacenamiento del 5 al 10\%, y participación en el mercado de un 5 al 15\% respecto a la producción nacional (MAGAP, 2016).

\section{Precio de una saca versus precio a nivel de consumidor}

Los resultados señalan un modelo de correlación lineal positivo de $0.961 \quad(p<0.05)$ entre el precio que recibe el productor al vender una saca de arroz $(90.70 \mathrm{~kg})$ y el precio a nivel del consumidor. Durante el período analizado se observa una leve disminución de la brecha entre los precios de origen y destino puesto que el precio al productor se incrementó en $146.70 \%$, mientras que el precio al consumidor aumentó en $123.40 \%$. Para Zanin y Piedade (2012), los cambios en los márgenes de comercialización de arroz son explicados especialmente por los choques de la demanda (precio al por menor) y de la oferta (precio al productor) y en menor medida por los costos de insumos agrícolas.

\section{Precio a nivel de consumidor versus consumo per cápita de arroz}

Los resultados indican una correlación lineal positiva de $0.7925(\mathrm{p}<0.05)$ entre precio a nivel de consumidor de arroz y consumo por habitante. El pronóstico del consumo per cápita para los próximos 12 años (Figura 6), muestra

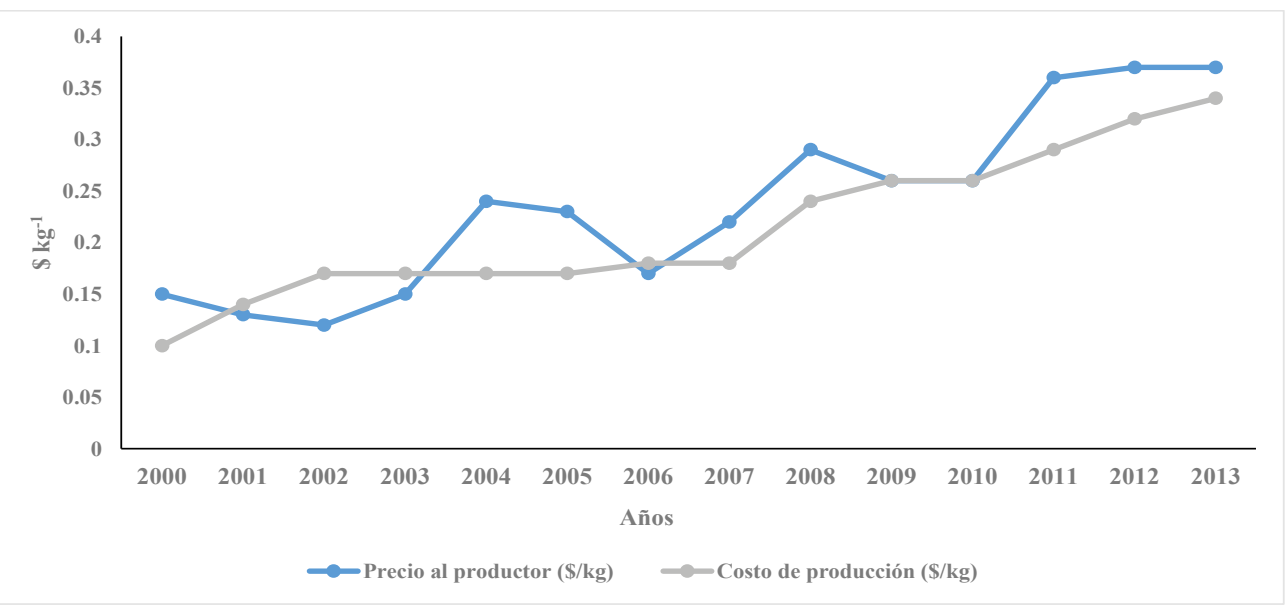

Figura 5. Precio al productor vs costo unitario de producción $\left(\$ \mathrm{~kg}^{-1}\right)$, periodo $2000-2013$

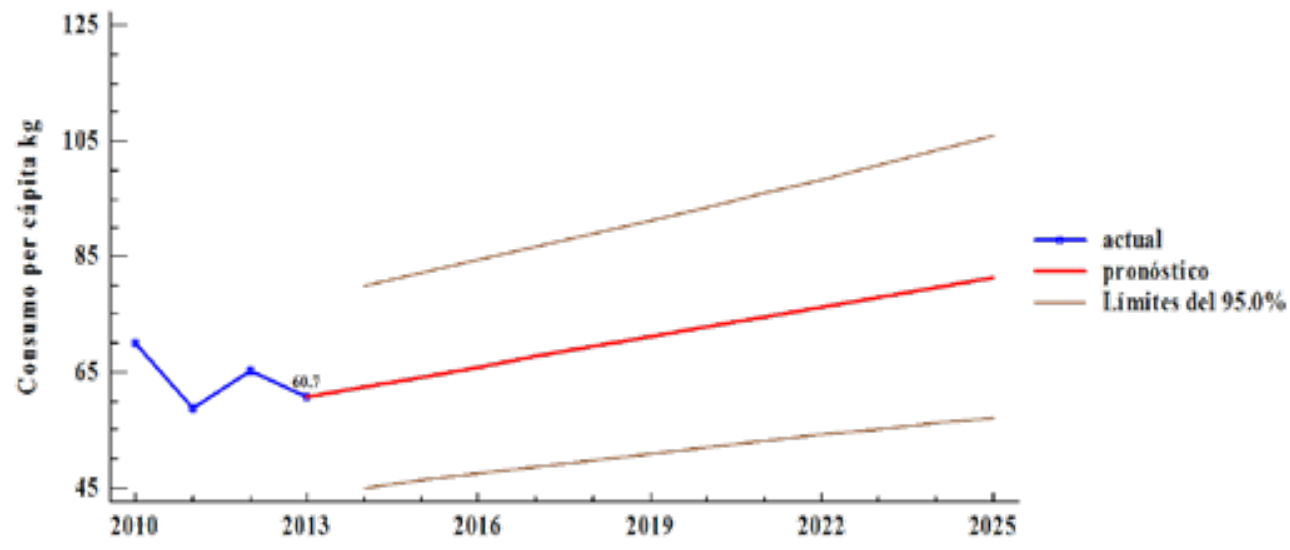

Figura 6. Pronósticos para consumo per cápita, periodo 2014-2025 
una tendencia lineal, con un promedio de $71.94 \mathrm{~kg}$, y rango entre 45.02 y $105.80 \mathrm{~kg}$ con límite inferior entre $45.02 \mathrm{y}$ 57.05 y superior entre 79.90 y $105.80 \mathrm{~kg}$. La predicción sobre consumo per cápita y precios de arroz se presentan también a nivel internacional, pues, según Ceballos y Pire (2015), al incrementarse el número de consumidores en países con economías emergentes como China, India y Brasil, se espera que el precio del arroz para los próximos años 2013 y 2014 tiendan al alza.

\section{Conclusiones}

E Ecuador, durante los años 2000 al 2013 se presenta una leve disminución de la brecha entre los precios de origen y destino puesto que el precio al productor se incrementó en $146.70 \%$, mientras que el precio al consumidor aumentó en $123.40 \%$.

El mejoramiento de los precios unitarios a nivel de agricultor son en parte explicados por el pago de precios mínimos de sustentación de arroz controlados por la UNAEP. Los precios del productor están correlacionados con los precios al consumidor, resaltando la variable precio por kilogramo de arroz al consumidor que fue superior en $226.10 \%$ al precio del productor, confirmándose la hipótesis de que coexisten situaciones de precios bajos en origen con altos precios en destino.

El precio del kilogramo de arroz para los próximos 12 años, fluctúa entre USD 0.40 y USD 0.50 para el productor y entre USD 1.13 y USD 1.38 a nivel de consumidor. Los precios de arroz a nivel de productor fueron superiores o iguales a los costos de producción durante 11 de los 14 años analizados. Los precios al consumidor están altamente relacionados con el consumo per cápita y mantienen la misma tendencia que el mercado externo.

\section{Bibliografía}

Alonso, J., Gallego, A. (2010). Integración de los precios en los canales minorista y mayorista arroz, papa y fríjol en la ciudad de Cali. Economía Gestión Desarrollo, 10: 7996.

Andrés, M., Alfaro, J., López V., (2012). Evolución de precios agrarios percibidos y pagados por el agricultor en España (2000-2009). Revista de la Facultad de Ciencias Agrarias UNCUYO, 44 (2): 1-13.

Artega, C., Granados, J., Ojeda, J. (2013). Determinantes de los precios internacionales de los bienes básicos. Ensayos sobre Política Económica, 31: 85-107.

Asamblea Nacional. (2011). Ley Orgánica de Regulación y Control del Poder de Mercado. Disponible en: www.planificacion.gob.ec/wp-content/uploads/ downloads/2012/10/content/uploads/downloads/2012/10/ Ley-Organica.pdf

Banco Central del Ecuador (BCE). (2015). Reporte de Coyuntura, sector agropecuario. Arroz de verano. Publicaciones técnicas, 87: 10-18.

Briceño, I., Álvarez, L. (2010). Evaluación de un sistema de preparación del suelo y siembra en el cultivo de arroz (Oriza sativa L). Rev. Unell. Cien. Tec., 28: 16-24.

Ceballos, S., Pire, R. (2015). Estimación del precio internacional del arroz (Oryza sativa L.) bajo el modelo ARIMA. Revista Mexicana de Ciencias Agrícolas Pub. Esp. 11: 2083-2089.

Cortez, L., Vargas, P. (2013). Arroz orgánico una alternativa para la seguridad alimentaria en la ciudad de Guayaquil. Yachana 2(1): 203-211.

Garcés, F., Díaz, T., Aguirre, A. (2012). Severidad de la quemazón (Pyricularia oryzae Cav.) en germoplasma de arroz F1 en la Zona Central del Litoral ecuatoriano. Ciencia y Tecnología, 5(2): 1-6.

Gavilánez, F., Martillo, J., Morán, C., Cruz, C., Martínez, F. (2016). Influencia del zinc sobre el estrés generado por la aplicación de una mezcla herbicida en el cultivo de arroz (Oryza sativa). El misionero del agro, 10(3): 8-17.

Indexmundi. Cereales. (2015). Arroz. Precios mensualDólares americanos por tonelada métrica. http://www. indexmundi.com/es/precios-de-mercado

Instituto Nacional de Investigaciones Agropecuarias. [INIAP]. (2013). Estación Experimental Litoral Sur. Informe Técnico Anual DNPV. [Versión electrónica interna].

Ministerio de Agricultura y Ganadería Acuacultura, Caza y Pesca [MAGAP]. (2014). Registro oficial. Órgano del Gobierno del Ecuador. Acuerdo $\mathrm{N}^{\circ}$ 119. https:// www.registroficial.gob.ec/index.php/publicaciones/ monthlyarchive/00/2014/limit,0.html?1ab2cf323282e6c $48 \mathrm{a} 950 \mathrm{a} 7352 \mathrm{cf} 474 \mathrm{~b}=1 \&$ task $=$

Ministerio de Agricultura y Ganadería, Acuacultura, Caza y Pesca [MAGAP]. (2015). Costos de producción, superficie sembrada, producción, rendimiento. 20002013. Dirección Provincial Agropecuaria.

Ministerio de Agricultura y Ganadería, Acuacultura, Caza y Pesca [MAGAP]. (2016). La política agropecuaria ecuatoriana: hacia el desarrollo territorial rural sostenible, 2015-2025. I Parte. Quito, Ecuador. 480 p.

Monasterio, C., Pierre, F., Barreto, T., Marin, C., Mora, O., Tablante, J., Maturet, W., Mendoza, C. (2011). Influencia del fenómeno El Niño/Oscilación del Sur sobre la precipitación y rendimiento del cultivo de maíz en el municipio Peña, estado Yaracuy. Agronomía Tropical, 61(1): 59-72.

Najar, C., Álvarez, J. (2007). Mejoras en el proceso productivo $\mathrm{y}$ modernización mediante sustitución y tecnologías limpias en un molino de arroz. Industrial Data, 10(1): 22-32. 
Sistema de Información Nacional de Agricultura, Acuacultura y Pesca. [SINAGAP]. (2015). Sector Agropecuario.

Tolentino, J. (2014). La producción de arroz del estado de Morelos: una aproximación desde el enfoque SIAL. Estudios sociales, 44(22): 39-61.

Vieira, R., Lindner, M., Branco, C. (2014). The ecological rice production in the Metropolitan Region of Porto Alegre: Settelements, rural development and socio territorial transformations. FSA, 12(1): 121-138.

Vivas, V., Albisu, L. (2011). Competitividad de la cadena arrocera del estado Portuguesa, Venezuela, en procesos de integración sudamericanos. Unell. Cienc. Tec., 29: 67-79.

Zanin, V., Piedade, M. (2012). Análise da margen de comercialização do arroz gaúcho no mercado de Sao Paulo após o Plano Real. Fundação de Economía e Estatistica, 1(108): 1-24. 